\title{
Perfil lipídico da dieta alimentar como fator de risco para doenças cardiovasculares em idosas ativas
}

\author{
The alimentary diet lipidic profile as factor of risk for cardiovascular diseases in \\ active aged woman

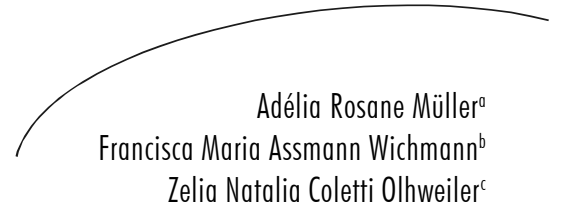

\section{Resumo}

O objetivo deste trabalho é avaliar o perfil lipídico da dieta e sua correlação com os fatores de risco para doenças cardiovasculares, em uma amostra de 130 mulheres acima de 60 anos. Para avaliar o estado nutricional utilizaram-se o Índice de Massa Corporal (IMC) e Circunferência da Cintura (CC), e a análise da dieta foi feita através do recordatório alimentar de 24 horas. Como resultados, observou-se que $65,4 \%$ da amostra encontram-se em sobrepeso, 32,3\% em eutrofia e 2,3\% com baixo peso. Quanto ao fator de risco, avaliado através da circunferência abdominal, constatou-se que 38,5\% das idosas estavam dentro da normalidade, $20,8 \%$ estavam em risco aumentado e $40,8 \%$ em risco muito aumentado, representando $61,6 \%$ da população estudada em risco para o desenvolvimento de doenças cardiovasculares. Identificou-se forte associação entre índice de massa corporal e circunferência abdominal nas idosas avaliadas $(\mathrm{p}<0,05)$. Avaliando-se a dieta alimentar das idosas, notou-se que, em média o consumo de calorias é de 1440,56 (+39,04), o consumo médio de lipídios totais e insaturados em relação ao valor energético total (VET) foram de $29,28 \%(+8,87)$ e $13,31 \%(+5,77)$ respectivamente. A média de colesterol alimentar foi de $156 \mathrm{mg}(+91,62)$ e de fibras, 16,84 g (+8,87). Faz-se necessária uma análise mais detalhada e intervenção eficaz para a promoção da melhora do perfil lipídico e da saúde geral dessa população.

\author{
Palavras-chave: \\ gorduras na dieta; \\ fatores de risco; \\ doenças \\ cardiovasculares; \\ mulheres; nutrição \\ do idoso; índice de \\ massa corporal; \\ circunferência \\ abdominal ; \\ consumo de \\ alimentos; \\ comportamento \\ alimentar $\square$
}

Correspondência / Correspondence

Adélia Rosane Müller

Condomínio Quintas do Sol, Qd 09, cj. E, lote 10, casa 02 - SHIS, Lago Sul

71680-370 - Brasília, DF, Brasil

E-mail: adeliamuller@terra.com.br 


\section{Abstract}

The aim of this study is to evaluate the diet lipidic profile and its relationship with cardiovascular diseases (DVC) risk factors in a sample of 130 women above 60 years. To evaluate the nutritional state, it was used the Body Mass Index (BMI) and the Waist Circumference (WC) and the analysis of the diet was made through last 24-hours meals remember. We observed that $65.4 \%$ of the sample was overweight, $32.3 \%$ was in a normal level and $2.3 \%$ indicated low weight. Related to the risk factors, that we evaluated through the abdominal circumference, $38.5 \%$ of the women were in a normal level, $20.8 \%$ were in an increased risk level and $40.8 \%$ in a very increased risk level, results that classificate $61.6 \%$ of the studied population risking to develop cardiovascular illnesses. We evidenced some strong association between the Body Mass Index and the Abdominal Circumference $(\mathrm{p}<0.05)$. Evaluating it alimentary diet of the aged ones, noticed that, on average the consumption of calories is of $1440,56(+39,04)$, the average consumption of total and insaturated fat in relation to the total energy value had been of $29,28 \%(+8,87)$ and $13.31 \%$ $(+5,77)$ respectively. The alimentary cholesterol average was of $156 \mathrm{mg}(+91,62)$ and staple fibres, $16,84 \mathrm{~g}(+8,87)$. An analysis some more detailed becomes necessary and efficient intervention looking for an improvement in the lipidic profile and the general health characteristics of this population

Key words: dietary fats ; risk factors; cardiovascular diseases; women ; elderly nutrition ; body mass index ; abdominal circumference; food consumption; feeding behavior

\section{INTRODUÇÃO}

$\mathrm{Na}$ medida em que mais pessoas vivem até idade bem avançada, aumenta a prevalência de doenças em que a idade é fator de risco, como por exemplo, as doenças cardiovasculares. Associado ao fator idade, percebe-se um alto índice de obesidade, inclusive na terceira idade, sendo necessário maior monitoramento do estado nutricional nessa faixa etária.

Conforme Tirapegui ${ }^{14}$ (2000), "o envelhecimento é caracterizado por uma série de modificações fisiológicas e psicológicas que estão relacionadas, por sua vez, com alterações no estado nutricional'.
Dentre as alterações fisiológicas associadas ao envelhecimento, que afetam a composição e o estado nutricional dos idosos, está a substituição progressiva da massa corpórea magra por gordura e tecido conjuntivo. Para os idosos, a nutrição é especialmente importante, em função de modificações sensoriais, gastrintestinais, metabólicas, neurológicas e ao desenvolvimento de doenças crônico-degenerativas, que interferem diretamente no consumo de alimentos.

Os idosos tendem a apresentar elevação da pressão arterial, do colesterol total e LDL (colesterol de baixa densidade) e diminuição do HDL (colesterol de alta densidade), principalmente quando associados à obesidade e sedentarismo. ${ }^{7}$ 
Sabe-se que as doenças cardiovasculares constituem a principal causa de mortalidade e as mesmas são influenciadas por um conjunto de fatores de risco, alguns modificáveis mediante alterações no estilo de vida, como a dieta e o exercício regular.

A ingestão excessiva de gordura pode contribuir para várias doenças, entre elas a obesidade e doenças cardiovasculares, que são responsáveis pela morte de grande parte da população idosa. ${ }^{5}$

O presente trabalho visou avaliar as variáveis antropométricas e dietéticas de $130 \mathrm{mu}$ lheres com 60 anos ou mais, a fim de descrever o perfil nutricional e alimentar das mesmas, relacionando-o com os fatores de risco para doenças cardiovasculares.

\section{METODOLOGIA}

Trata-se de um estudo descritivo com dados retrospectivos, coletados pelo projeto "Ações para o Envelhecimento com Qualidade de Vida", da Universidade de Santa Cruz do Sul. Os sujeitos freqüentam as sessões de hidroginástica na Instituição três vezes por semana, 50 minutos por sessão.

As variáveis utilizadas no estudo são: peso, estatura, idade, sexo, circunferência da cintura e quantidade de lipídios da dieta alimentar, analisados quanto ao percentual em relação ao Valor Energético Total (VET).

Os indicadores utilizados para a avaliação do estado nutricional das idosas são o índice de massa corporal, a circunferência abdominal e o teor de lipídios presentes na dieta alimentar. Os sujeitos foram divididos de acordo com a faixa etária - de 60 a 69 anos, de 70 a 79 anos e acima de 80 anos de idade-, para uma análise mais detalhada da interferência do estado nutricional nas condições de saúde dessa população.

Para a aferição da estatura, utilizou-se um estadiômetro transportável, da marca Cardiomed, com $212 \mathrm{~cm}$ e precisão de $0,1 \mathrm{~cm}$. Solicitou-se que as pacientes tirassem os calçados e se colocassem de costas para o estadiômetro, em posição ereta e com os pés unidos. $^{2}$

A verificação do peso corporal, por sua vez, foi realizada em uma balança digital Plenna, com capacidade de $200 \mathrm{Kg}$ e com precisão de 100g. Pediu-se que as mesmas retirassem os sapatos e objetos ou roupas pesadas. Instruiu-se para que ficassem em pé de frente para a escala da balança, com afastamento lateral dos pés, eretos e com olhar fixo para a frente. $^{2}$

Para a medida da circunferência da cintura, utilizou-se uma fita métrica flexível com precisão de $1 \mathrm{~mm}$, da marca Cardiomed. A medição foi realizada com a paciente em pé, circundando-se a fita na linha natural da cintura, na região mais estreita entre o tórax e o quadril, no ponto médio entre a última costela e a crista ilíaca. A leitura foi realizada no momento da expiração. ${ }^{2}$

Concernente a análise da dieta, aplicou-se o método Recordatório Alimentar de 24 ho- 
ras, no qual idosas foram instigadas a recordar todos os alimentos e bebidas consumidos no dia anterior, quantificados em medidas caseiras e anotados no prontuário. Utilizou-se um mostruário de utensílios básicos de cozinha, para dimensionar o tamanho da porção e a média per capita. Os dados obtidos através do Recordatório Alimentar de 24 horas foram processados no Programa de Apoio à Nutrição Virtual Nutri, produzido pela Faculdade de Saúde Pública da Universidade de São Paulo. Na análise da ingestão de lipídios, os resultados foram comparados com a recomendação da Framingham Heart Study, que preconiza valores iguais ou inferiores a 30\% do valor calórico total, e um máximo de $10 \%$ advindos de gordura saturada. ${ }^{5}$

Utilizou-se, para a análise antropométrica do estado nutricional, o Índice de Massa Corporal (IMC), calculado a partir da divisão da massa corporal em quilogramas pela estatura elevada ao quadrado $\left(\mathrm{kg} / \mathrm{m}^{2}\right)$ e os pontos de corte recomendados pelo Ministério da Saú$\operatorname{de}^{10}$ (MS, 2004) para avaliação da população idosa. Com base no IMC, foram considerados baixo-peso o IMC $<22$ e sobrepeso o IMC $>27 \mathrm{~kg} / \mathrm{m}^{2}$.

A verificação do risco de desenvolvimento de complicações associadas à obesidade, através da circunferência da cintura, tomou por base os valores sugeridos por sexo pela Organização Mundial da Saúde, em 1998 (apud Cuppari ${ }^{2}$ 2002), que considera que, para mulheres, valores acima de $80 \mathrm{~cm}$ representam um risco elevado e acima de $88 \mathrm{~cm}$ um risco muito elevado.
A análise dos dados foi processada no programa Statistical Package for the Social Science (SPSS), versão 9.0 for Windows, por meio de análise descritiva e comparativa com estudos da literatura, considerando-se um nível de significância de p<0,05.

A significância estatística das diferenças de médias antropométricas e dietéticas entre as faixas etárias foram verificadas por meio do teste t-student. Foram realizadas a análise de variância (ANOVA) e a comparação de Tukey's para verificar o efeito da idade nas variáveis antropométricas.

\section{RESULTADOS}

Foram avaliadas 130 mulheres com média de idade de 67,8 anos. A idade mínima verificada na amostra foi 60 anos e a máxima 85 , sendo $66,9 \%(\mathrm{~N}=87)$ na faixa etária de 60 a 69 anos, 30,8\% (N=40) de 70 a 79 anos e $2,3 \%(\mathrm{~N}=3)$ acima de 80 anos.

\section{Variáveis antropométricas}

Quando avaliadas as variáveis peso e estatura, verificou-se como peso mínimo 44,20 $\mathrm{kg}$, sendo o máximo 106,60 kg, com média $71,70 \mathrm{~kg}$ e desvio padrão 11,55. Já para a estatura, o menor valor encontrado foi de 142 $\mathrm{cm}$, o maior valor foi $174 \mathrm{~cm}$, com média de $156,84 \mathrm{~cm}$ e desvio padrão 6,35 .

Em relação ao estado nutricional das idosas, conforme pode ser observado no Gráfico 1, verificou-se que a prevalência 
geral de sobrepeso corresponde a $65,4 \%$, $\operatorname{com} 32,3 \%$ da amostra em eutrofia e apenas $2,3 \%$ das idosas na condição de baixo-peso.
O estado nutricional da amostra, segundo grupo etário, é apresentado na tabela $1 . \mathrm{O}$ valor mínimo de IMC encontrado foi 19,85 $\mathrm{kg} / \mathrm{m}^{2}$ e o máximo foi $41,35 \mathrm{~kg} /{ }^{2}$, a média

Gráfico 1 - Estado nutricional das idosas

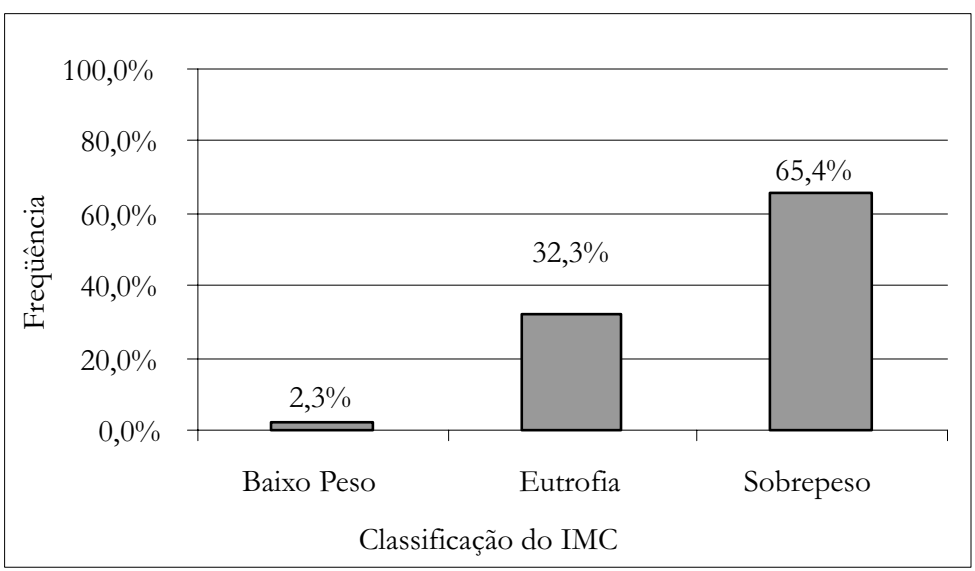

Tabela 1 - Estado nutricional das idosas, segundo faixa etária

\begin{tabular}{lcccccr}
\hline Faixa etária & \multicolumn{9}{c}{ Estado nutricional } \\
\hline & \multicolumn{2}{c}{ Baixo peso $^{1}$} & \multicolumn{2}{c}{ Eutrofia $^{2}$} & \multicolumn{2}{c}{ Sobrepeso $^{3}$} \\
\cline { 2 - 7 } & $\mathrm{N}$ & $\%$ & $\mathrm{~N}$ & $\%$ & $\mathrm{~N}$ & $\%$ \\
$60-69$ anos & 3 & 2,3 & 30 & 23,1 & 54 & 41,5 \\
$70-79$ anos & & - & 11 & 8,5 & 29 & 22,3 \\
80 anos ou + & 3 & - & 1 & 0,8 & 2 & 1,5 \\
Total & & 2,3 & 42 & 32,3 & 85 & 65,4 \\
\hline
\end{tabular}

${ }^{1} \mathrm{IMC}<22 \mathrm{~kg} / \mathrm{m}^{2} ; \quad{ }^{2} \mathrm{imc}>22<27 \mathrm{~kg} / \mathrm{m}^{2} ;{ }^{3} \mathrm{imc}>27 \mathrm{~kg} / \mathrm{m}^{2}$

foi $29,15 \mathrm{~kg} / \mathrm{m}^{2}$ e o desvio padrão foi de 4,48. Os valores mínimos e máximos de IMC foram verificados na faixa etária de 60 a 69 anos, possivelmente por ser grupo predomi- nante da amostra. $O$ valor mínimo verificado para a circunferência da cintura foi de $60 \mathrm{~cm}$, o máximo de $123 \mathrm{~cm}$, a média foi $92,49 \mathrm{~cm}$ e desvio padrão de 10,69. 
Analisando-se o estado nutricional por faixas de idades, observamos que $41,5 \%$ das mulheres com sobrepeso encontram-se na faixa etária dos 60 a 69 anos - na qual também encontramos o maior índice de risco muito aumentado para doenças cardiovasculares.

Avaliando-se o risco cardiovascular geral e por faixas etárias (tabela 2), observamos que $40,8 \%$ do total das idosas apresentam risco muito aumentado para doenças cardiovasculares, sendo $26,9 \%$ na faixa etária dos 60 a 69 anos. Dentro da faixa de normalidade encon- tramos $38,5 \%$ das idosas, tendo $27,7 \%$ de 60 e 69 anos. Já na faixa de risco aumentado, encontramos $20,8 \%$ das idosas, tendo $12,3 \%$ de 60 a 69 anos. O que nos preocupa realmente com esses dados é o fato de que mais da metade das idosas avaliadas $(61,6 \%)$ apresenta risco aumentado ou muito aumentado de doenças cardiovasculares, pois, segundo estudo de Cabrera e Filho ${ }^{1}$ (2001) sobre a prevalência, distribuição e associação com hábitos e comorbidades, há maior prevalência de hipertensão arterial e diabetes mellitus em idosos apresentando circunferência da cintura aumentada.

Tabela 2 - Risco cardiovascular conforme faixa etária

\begin{tabular}{lcrcrcr}
\hline Faixa etária & \multicolumn{5}{c}{ Risco cardiovascular } \\
\hline & \multicolumn{2}{c}{ Normal } & \multicolumn{2}{c}{ Risco aumentado } & \multicolumn{2}{c}{ Risco muito aumentado } \\
& $\mathrm{N}$ & $\%$ & $\mathrm{~N}$ & $\%$ & $\mathrm{~N}$ & $\%$ \\
\cline { 2 - 7 } $60-69$ anos & 36 & 27,7 & 16 & 12,3 & 35 & 26,9 \\
$70-79$ anos & 13 & 10 & 11 & 8,5 & 16 & 12,3 \\
80 anos ou + & 1 & 0,8 & & & 2 & 1,5 \\
Total & 50 & 38,5 & 27 & 20,8 & 53 & 40,8 \\
\hline
\end{tabular}

Verificou-se forte associação entre IMC e CC nas idosas pesquisadas ( $\mathrm{p}<0,05)$, sendo que $60 \%$ da população avaliada encon- tram-se com sobrepeso e risco muito aumentado para doenças cardiovasculares (tabela 3). 
Tabela 3 - Classificação das variáveis antropométricas índice de massa corporal (IMC) e circunferência da cintura (CC), das idosas

\begin{tabular}{|c|c|c|c|c|c|c|}
\hline & & \multicolumn{5}{|c|}{ Classificação ${ }^{*} \mathrm{CC}$} \\
\hline & & & $N^{*}$ & $\mathrm{~A}^{*}$ & MA* & Total \\
\hline \multirow[t]{6}{*}{ *IMC } & Baixo peso & count & 3 & - & - & 3 \\
\hline & & $\%$ IMC & $100 \%$ & - & - & $2,3 \%$ \\
\hline & Eutrófico & count & 32 & 8 & 2 & 42 \\
\hline & & $\%$ IMC & $76,2 \%$ & $19 \%$ & $4,8 \%$ & $32,3 \%$ \\
\hline & Sobrepeso & count & 15 & 19 & 51 & 51 \\
\hline & & $\%$ IMC & $17,6 \%$ & $22,4 \%$ & $60 \%$ & $65,4 \%$ \\
\hline \multirow[t]{2}{*}{ Total } & & count & 50 & 27 & 53 & 130 \\
\hline & & $\%$ IMC & $38,5 \%$ & $20,8 \%$ & $40,8 \%$ & $100 \%$ \\
\hline
\end{tabular}

IMC: índice de massa corporal; ${ }^{*}$ CC: circunferência da cintura.

$\mathrm{N}^{*}$ - normal; $\mathrm{A}^{*}$ - risco aumentado; $\mathrm{MA}^{*}$ - risco muito aumentado.

\section{Dieta alimentar}

Realizando a avaliação da dieta alimentar das idosas, nota-se que, em média, consumiram-se 1440,56 $(+39,04)$ calorias, variando entre 611,64 kcal e 3213,17 kcal (tabela 4).

Quanto ao consumo de gordura total e insaturada, ambas medidas como percentual do VET, os valores médios encontrados fo$\operatorname{ram} 29,28 \%(+8,87)$ e $13,31 \%(+5,77)$, respectivamente. O consumo de gordura total variou entre $9,99 \%$ e $53,67 \%$, enquanto que a insaturada apresentou valores entre $3,32 \% \mathrm{e}$ $27,67 \%$ (tabela 4). A quantidade média de colesterol observada foi de $156 \mathrm{mg}(+91,62)$, e seus valores mínimos e máximos foram de $15,40 \mathrm{mg}$ e $523,06 \mathrm{mg}$.

Tabela 4 - Mediana das quantidades de valor energético total (VET), lipídio total, lipidios insaturados, colesterol e fibras ingeridas pelas idosas

\begin{tabular}{lccccc}
\hline & VET ingerido & \% de lipídios & $\begin{array}{c}\text { \% de lipídios } \\
\text { insaturados }\end{array}$ & $\begin{array}{c}\text { Colesterol } \\
\text { ingerido (mg) }\end{array}$ & $\begin{array}{c}\text { Fibras } \\
\text { ingeridas }(\mathrm{g})\end{array}$ \\
\hline Média & 1443,92 & 29,28 & 13,31 & 156,00 & 16,84 \\
Desvio padrão & 439,05 & 8,87 & 5,77 & 91,62 & 7,95 \\
Valor mínimo & 611,64 & 9,99 & 3,32 & 15,40 & 3,99 \\
Valor máximo & 3213,17 & 53,67 & 27,67 & 523,14 & 59,06 \\
Recomendações & 1900,00 & $20-30 \%$ & $20 \%$ & $<200 \mathrm{mg}$ & $20-30 \mathrm{~g}$ \\
\hline
\end{tabular}

*VET: valor energético total. 
Embora o programa utilizado para a análise dietética não forneça a quantidade de gordura saturada consumida, estes podem ser presumidos subtraindo-se da gordura total a quantidade de gordura insaturada. No grupo estudado, observou-se um consumo de gordura saturada próximo a $17 \%$, sendo que a recomendação é de que não devem ultrapassar 10\% do VET.

Vale ressaltar que a ingestão excessiva de gordura pode contribuir para várias doenças, entre elas a obesidade e doenças cardiovasculares, que são responsáveis pela morte de grande parte da população idosa. ${ }^{5}$

Com relação à ingestão diária de fibras alimentares, o valor médio observado foi de $16,84 \mathrm{~g}(+8,87)$ e sua variação foi de $3,99 \mathrm{~g} \mathrm{a}$
59,06 g (tabela 4). A média é considerada baixa, face à importância das fibras na prevenção de DCVs.

Ao se avaliar a prevalência dos fatores de risco para as doenças cardiovasculares na população estudada, verificou-se (tabela 5), que $89,2 \%$ se encontram em risco, $65,4 \%$ possuem alteração no índice de massa corporal, e, aliados aos fatores de riscos antropométricos, encontramos $73,8 \%$ com uma ingesta reduzida em fibras alimentares, $20,8 \%$ com alta ingestão em lipídeos na dieta alimentar e 17,7\% com ingestão elevada de colesterol na dieta alimentar. Destaca-se que nesta parcela da população identificamos tanto os riscos antropométricos como os riscos dietéticos para o desenvolvimento das doenças cardiovasculares.

Tabela 5 - Prevalência dos fatores de risco estudados para as doenças cardiovasculares

\begin{tabular}{lcccc}
\hline \multicolumn{1}{c}{ Indicadores } & \multicolumn{2}{c}{ Sem risco } & \multicolumn{2}{c}{ Com risco } \\
\hline \multirow{2}{*}{ IMC } & $\mathrm{N}$ & $\%$ & $\mathrm{~N}$ & $\%$ \\
CCC & 35 & 34,6 & 85 & 65,4 \\
Fibras & 14 & 10,8 & 116 & 89,2 \\
Lipidios & 34 & 26,2 & 96 & 73,8 \\
Colesterol & 103 & 79,2 & 27 & 20,8 \\
\hline
\end{tabular}

Assim, nota-se que a alimentação da população em questão apresenta-se inadequada quantitativamente, o que se reflete no estado fisiológico encontrado, evidenciando o aumento do risco cardiovascular.

\section{DISCUSSÃO}

De modo geral, os resultados obtidos confirmam outros estudos, demonstrando a prevalência de sobrepeso em mulheres idosas, que 
representa alto risco para doenças cardiovasculares.

Santos e Sichieri ${ }^{13}$ (2005) relataram, em seu estudo sobre IMC e indicadores antropométricos de adiposidade em idosos, uma maior proporção de sobrepeso e de inadequação de gordura em mulheres e prevalência de apenas 3,5\% de magreza. Dados do IBGE (2004) também apontam para uma maior prevalência de excesso de peso na população brasileira em todas as regiões, especialmente nas mulheres da região Sul e Sudeste. Filippsen ${ }^{4}$ (2002) observou excesso de adiposidade em $80 \%$ de sua amostra, ocorrendo muito mais entre as mulheres $(91,7 \%)$ do que entre os homens (37,8\%). Vários outros estudos apontam a mesma tendência de elevação de sobrepeso em mulheres idosas mais do que em homens (Cabrera e Jacob Filho ${ }^{1}$, 2001; Sampaio e Figueiredo $^{12}$, 2005; Marques $^{8}$ et al., 2005).

Sampaio e Figueiredo ${ }^{12}$ (2005) e Cabrera e Jacob Filho ${ }^{1}$ (2001), ao correlacionarem IMC com CC, encontraram características semelhantes, mostrando a evidente associação entre estas variáveis.

Através da avaliação da dieta das idosas, podemos observar uma inadequação na proporção de gorduras saturadas e insaturadas e um baixo consumo de fibras, reiterando a afirmação de que a população estudada encontra-se em risco para doenças cardiovasculares. Verifica-se a necessidade de avaliar nessa dieta as quantidades de carboidratos e proteínas ingeridas, que foram desconsiderados por não fazerem parte dos objetivos deste estudo.
Conforme Teixeira Neto (2003), reduções, mesmo modestas, da ingestão de gorduras saturadas têm um impacto benéfico na evolução das doenças cardiovasculares.

A obesidade, de modo geral, tem forte associação com a presença de fatores de risco cardiovascular, aqui avaliados através da medida da circunferência da cintura. Os resultados encontrados nos permitem dizer que as idosas estudadas apresentam grande propensão para desenvolver doenças crônicas não-transmissíveis, dentre elas, doenças cardiovasculares.

Devemos levar em conta que as idosas pesquisadas praticam atividade física (hidroginástica) com regularidade, que é considerado como fator de proteção cardiovascular e forte indicador de boa qualidade de vida. Embora existam poucas pesquisas que definam as necessidades nutricionais específicas para idosos ativos, sabe-se que os indivíduos idosos, de forma geral, devem ter uma dieta variada, saudável e balanceada. As recomendações dietéticas diárias são as mesmas aplicadas a indivíduos saudáveis, e não levam em conta o nível de atividade física.

A prevalência de sobrepeso observada nas mulheres idosas, ao lado dos achados relativos aos fatores de risco cardiovascular, aponta para a necessidade imperiosa de promoção do adequado estado nutricional, de prevenção e controle de obesidade e uma maior atenção para a saúde do idoso.

Existem ainda poucos estudos realizados referentes ao estado nutricional e análise de 
ingesta e hábitos alimentares de idosos. Portanto, recomenda-se a realização de mais pesquisas englobando esse grupo populacional, a fim de servir como base de dados para a implementação de adequadas políticas públicas e monitoramento desta importante parcela da sociedade em visível crescimento.

A adequada nutrição, associada à prática de atividade física regular durante o processo do envelhecimento, é componente primordial para a manutenção da saúde nesse ciclo da vida, havendo a necessidade de mais investimentos em prevenção e monitoramento, a fim de garantir o envelhecimento saudável.

\section{NOTAS}

a,b,c Universidde Federal de Santa Cruz do Sul, Departamento de Educação Física e Saúde, Santa Cruz do Sul, RS, Brasil

a Nutricionista, E-mail: adeliamuller@terra.com.br

b Nutricionista, Mestre e Doutoranda em Desenvolvimento Regional

E-mail: francis@unisc.br

c Educadora Física, Mestre em Ciências do Movimento E-mail: zelia@unisc.br

\section{REFERÊNCIAS}

1. Cabrera MAS, Jacob Filho W. Obesidade em idosos: prevalência, distribuição e associação com hábitos e comorbidades. Arq Bras Endocrinol Metabol 2001 out [acesso 2005 out 10]; 45(5). Disponível em: URL: http://www.scielo.br.

2. Cuppari L, organizador. Guia de nutrição: nutrição clínica do adulto. São Paulo: Manole; 2002. 406 p.
3. Figueroa JCG, Frank AA. Nutrição e atividade física para a promoção de saúde no envelhecimento. Revista digital 2002 maio [acesso 2005 out 30] ; 8 (48). Disponível em: URL: http://www.efdeportes.com

4. Filippsen EK. Estado nutricional de idosos institucionalizados e não institucionalizados residentes em Londrina, PR. [dissertação]. São Paulo: Faculdade de Ciências Farmacêuticas, Programa de Pós-Graduação em Ciência dos Alimentos; 2002. 107 p.

5. Frank AA, Soares EA. Nutrição no envelhecer. São Paulo: Atheneu; 2004. 300 p.

6. Instituto Brasileiro de Geografia e Estatística IBGE. Pesquisa de orçamento familiar 20022003: Análise da disponibilidade domiciliar de alimentos e do estado nutricional no Brasil. Rio de Janeiro, 2004.

7. Mahan LK, Escott-Stump SE. Krause: alimentos, nutrição e dietoterapia. São Paulo: Roca, 2002. 1157 p.

8. Marques APO, et al. Consumo alimentar em mulheres idosas com sobrepeso. Textos sobre envelhecimento 2005 [acesso 2005 set. 03]; 8(2). Disponível em: URL: http://www.unati. uerj.br.

9. _ Prevalência de obesidade e fatores associados em mulheres idosas. Arq Bras Endocrinol Metabol 2005 jun [acesso 2005 nov 10]; 49(3). Disponível em: URL: http:/ / www.scielo.br

10. Ministério da Saúde. Vigilância Alimentar e Nutricional -SISVAN. Orientações básicas para a coleta, o processamento, a análise de dados e a informação em serviços de saúde. Brasília, 2004. CD ROM.

11. Sampaio LR. Avaliação nutricional e envelhecimento. Revista de nutric'aDo da PUCCAMP 2004 out-dez [acesso 2005 out 1]; 17: 507-14. Disponível em: URL: http:// www.scielo.br 
12. Sampaio LR, Figueiredo VC. Correlação entre o índice de massa corporal e os indicadores de distribuição de gordura corporal em adultos e idosos. Revista de nutric'abo da PUCCAMP 2005 jan-fev [acesso 2005 nov 10]: 18(1). Disponível em: URL: http:// www.scielo.br

13. Santos DM, Sichieri R. Índice de massa corporal e indicadores antropométricos de adiposidade em idosos. Rev Saúde Pública 2005; 39(2): 163-8.
14. Tirapegui J. Nutrição: fundamentos e aspectos atuais. São Paulo: Atheneu, 2000. 284p.

Recebido em : 24/8/2006

Aceito: 05/2/2007 
1 